\title{
Neonatal BCG Immunization Followed by DNAhsp65 Boosters: Highly Immunogenic but not Protective Against Tuberculosis - a Paradoxical Effect of the Vector?
}

\author{
A. C. Pelizon*, D. R. Martins*, S. F. G. Zorzella-Pezavento*, J. Seger*, L. A. Justulin Jr†, D. M. da

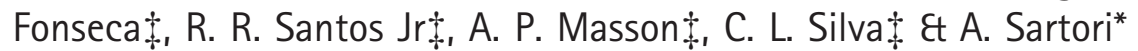

\begin{abstract}
*Department of Microbiology and Immunology, Biosciences Institute, São Paulo State University (UNESP), Botucatu, São Paulo, Brazil; †Department of Morfology, Biosciences Institute, São Paulo State University (UNESP), Botucatu, São Paulo, Brazil; and †Department of Biochemistry and Immunology, University of São Paulo (USP), Ribeirão Preto, São Paulo, Brazil
\end{abstract}

Received 24 July 2009; Accepted 13 November 2009

Correspondence to: A. Sartori, Department of Microbiology and Immunology, Biosciences Institute, São Paulo State University (UNESP), Distrito de Rubião Júnior s/n, CEP 18618-000, Botucatu, São Paulo, Brazil. E-mail: sartori@ ibb.unesp.br

\begin{abstract}
A new tuberculosis vaccine is urgently needed. Prime-boost strategies are considered very promising and the inclusion of BCG is highly desirable. In this investigation, we tested the protective efficacy of BCG delivered in the neonatal period followed by boosters in the adult phase with a DNA vaccine containing the hsp65 gene from Mycobacterium leprae (pVAXhsp65). Immune responses were characterized by serum anti-hsp65 antibody levels and IFN- $\gamma$ and IL-5 production by the spleen. Amounts of these cytokines were also determined in lung homogenates. Protective efficacy was established by the number of colony-forming units (CFU) and histopathological analysis of the lungs after challenge with Mycobacterium tuberculosis. Immunization with BCG alone triggered a significant reduction of CFU in the lungs and also clearly preserved the pulmonary parenchyma. BCG priming also increased the immunogenicity of pVAXhsp65. However, boosters with pVAXhsp65 or the empty vector abolished the protective efficacy of BCG. Also, higher IL-5 levels were produced by spleen and lungs after DNA boosters. These results demonstrated that neonatal BCG immunization followed by DNAhsp65 boosters is highly immunogenic but is not protective against tuberculosis.
\end{abstract}

\section{Introduction}

One-third of the world's population is infected with Mycobacterium tuberculosis and the disease caused by this pathogen is the leading cause of death from an infectious disease [1]. Factors such as co-infection with human immunodeficiency virus and emergence of drug resistance in $M$. tuberculosis strains added more burden to tuberculosis (TB) treatment [2]. The only licensed vaccine against $\mathrm{TB}$ is the attenuated Mycobacterium bovis Bacillus Calmette-Guérin (BCG) that is recommended by the World Health Organization for all infants under 1 year of age. Around 100 million newborn children are given this vaccine and the global vaccine coverage is estimated to be $80 \%$ [3]. In spite of this extensive use, large numbers of well-documented trials showed extensive variation, from 0 to $80 \%$, in BCG protective efficacy [4]. This has been attributed to variability in BCG vaccine strains, to expo- sure to environmental mycobacteria as well as host genetic characteristics [5, 6]. Although the overall efficacy is low, most studies agree that BCG protects against disseminated disease in newborns and children but that this immunity wanes with age, resulting in no or insufficient protection against adult pulmonary $\mathrm{TB}[7,8]$. In this context, there is great interest in the development of new vaccines against tuberculosis. A number of alternative living and non-living putative TB vaccines are being studied and discussed by many authors [9, 10]. Experimental evidences have indicated that DNA vaccines, because of their ability to induce a strong Th1 type of response, could contribute to TB control. DNA constructs encoding mycobacterial antigens such as the 65$\mathrm{kDa}$ heat shock protein (hsp65), Ag85A, Ag85B and PstS3 induced significant protective immunity [11, 12]. In addition, attempts to improve BCG by administering lower doses, by enhancing viability of BCG through 
improved lyophilization procedures, oral delivery and prime-boost protocols are being explored [13-15]. Safe and effective heterologous prime-boost regimens, which boosts or augments BCG or rBCG, is being considered the most realistic strategy for future TB control through immunization [8].

As a new TB vaccine will be administered to human neonates, it must be realized that neonates and young infants, as well as newborns from numerous animal species including mice, show particular limitations in generating protective immune responses. Neonatal murine immunization models using conventional vaccine antigens (measles, tetanus toxoid) in $\mathrm{BALB} / \mathrm{c}$ mice, gave responses similar to those found early in human infants. Early life B cell responses generally resulted in a slower and weaker increase of vaccine antibodies compared with adult mice. Furthermore, analyses of T-cell responses to these conventional vaccines indicated that early life $\mathrm{T}$ cell differentiation was preferentially polarized towards a Th2 pattern [16]. As a consequence of this Th2 bias, there is a deficiency in the induction of IFN- $\gamma, \mathrm{TNF}-\alpha$ and cytotoxic $\mathrm{T}$-cell responses that are essential for protection against many intracellular pathogens, including $M$. tuberculosis.

Researchers from our group previously demonstrated that a DNA plasmid encoding the Mycobacterium leprae $65-\mathrm{kDa}$ heat shock protein displayed both prophylactic and therapeutic effects in a murine model of tuberculosis $[17,18]$. As these experiments were all performed with adult $\mathrm{BALB} / \mathrm{c}$ mice, we investigated the protective efficacy of a prime-boost strategy initiated at the neonatal period. Neonate mice were primed with BCG and boosted in the adult phase with a DNA vaccine containing the hsp65 gene from $M$. leprae (pVAXhsp65). Even though priming with BCG determined an increased immune response to pVAXhsp65, this strategy decreased the protective effect of BCG.

\section{Materials and methods}

Animals. BALB/c mice were bred in the Animal Facility of São Paulo State University (UNESP), at the Biosciences Institute, and were used at 5 days (neonates) or 4 weeks of age (young adults). The animals were fed on a standard pellet diet and water ad libitum. The animal protocols used in this work were approved by the local ethical committee according to the guidelines adopted by the Brazilian College of Animal Experimentation.

Experimental design. Neonate BALB/c mice were immunized with BCG and boosted in the adult phase with two doses of pVAXhsp65 (BCG/vaccine group). The following control groups were included: BCG (neonate mice immunized with BCG only), BCG-vector (neonate mice immunized with BCG and boosted later with the empty vector), PBS/vector and PBS/vaccine (mice injected in the adult phase with two doses of the vector or the vaccine, respectively). Two weeks after the last DNA dose, the animals were either sacrificed and used for immune response analysis or challenged with $10^{5} \mathrm{CFU}$ of $M$. tuberculosis $\mathrm{H} 37 \mathrm{Rv}$ by the intratracheal route to analyse the protective efficacy of the vaccination strategy. Anti-hsp65 antibodies were evaluated in individual sera and cytokines (IFN- $\gamma$ and IL-5) were quantified in spleen cell culture supernatants and also in lung homogenates. Vaccination efficacy was evaluated by CFU determination and histopathological lung analysis.

Genetic vacine construction. The vaccine pVAXhsp65 was derived from the pVAX vector (Invitrogen ${ }^{\circledR}$, Carlsbad, CA, USA), previously digested with BamHI and NotI (Gibco BRL, Gaithersburg, MD, USA) by inserting a 3.3-kb fragment corresponding to the M. leprae hsp65 gene and the CMV intron $\mathrm{A}$. The empty pVAX vector was used for control. DH5 $\alpha$ Escherichia coli transformed with plasmid pVAX or the plasmid carrying the hsp65 gene ( $\mathrm{pVAXhsp65)}$ was cultured in LB liquid medium (Gibco BRL) containing ampicillin $(100 \mu \mathrm{g} / \mathrm{ml})$. The plasmids were purified using the Concert High Purity Maxiprep System (Gibco BRL). Plasmid concentrations were determined by spectrophotometry at $\lambda=260$ and $280 \mathrm{~nm}$ by using the Gene Quant II apparatus (Pharmacia Biotech, Buckinghamshire, UK).

Immunization procedures. Neonate mice were injected with BCG of the Moreau strain $\left(10^{5}\right.$ bacilli) administered by the intradermal route at the base of the tail. Boosters in the adult phase were administered with pVAXhsp65 $(100 \mu \mathrm{g} / 100 \mu \mathrm{l})$ in $25 \%$ saccharose delivered in the quadriceps muscle. Two pVAXhsp65 doses were administered at 15 days interval. Control animals received BCG during the neonatal period or two DNA doses (pVAXhsp65 or pVAX) in the adult phase.

Anti-hsp65 antibody levels. Sera were collected by retroorbital bleeding 2 weeks after the last DNA dose and anti-hsp65 specific antibody levels were evaluated by enzyme-linked immunosorbent assay (ELISA). Briefly, 96-well plates (Maxisorp Nunc-Immuno plates) were coated with $0.1 \mathrm{ml}$ of purified recombinant hsp65 $(5 \mu \mathrm{g} / \mathrm{ml})$ in coating solution $\left(14.3 \mathrm{~mm} \mathrm{Na}_{2} \mathrm{CO}_{3}\right.$, $10.3 \mathrm{~mm} \mathrm{NaHCO}, \mathrm{pH}$ 9.6), incubated at $4{ }^{\circ} \mathrm{C}$ overnight and then blocked with $10 \%$ fetal calf serum (FCS) in PBS for $60 \mathrm{~min}$ at $37^{\circ} \mathrm{C}$. Diluted serum samples (1:25) were tested. After incubation for $2 \mathrm{~h}$ at $37^{\circ} \mathrm{C}$, monoclonal antibody rat anti-mouse IgG1 and IgG2a biotinylated conjugates (A85-1 and R19-15, respectively, PharMingen), were added for detection of specific antibodies. After washing, plates were incubated at room temperature for $30 \mathrm{~min}$ with StreptAB kit (Dako, Carpinteria, CA, USA) and then revealed by adding $\mathrm{H}_{2} \mathrm{O}_{2}+$ OPD. Colour development was stopped by using $\mathrm{H}_{2} \mathrm{SO}_{4}$, and optical density was measured at $492 \mathrm{~nm}$.

CFU determination. Four weeks after infection with M. tuberculosis the bacterial load in the lungs was 
evaluated. Briefly, the organs were washed with sterile PBS and each one was placed in a Petri dish containing incomplete RPMI 1640 medium (Sigma). Lungs were fragmented and transferred to a conical tube containing digestion solution, prepared with Liberase Blendzyme 2 (Roche, Indianapolis, IN) diluted $(0.5 \mu \mathrm{g} / \mathrm{ml})$ in incomplete RPMI-1640. Samples were incubated at $37^{\circ} \mathrm{C}$ under agitation for $30 \mathrm{~min}$. After incubation, the cells were dispersed by using a 10-ml syringe and pelleted by centrifugation for $10 \mathrm{~min}$ at $400 \mathrm{~g}$. Cells were then washed with complete RPMI 1640, passed through a Nytex screen (Sigma) and resuspended in complete RPMI 1640. Serial dilutions were plated on supplemented $7 \mathrm{H} 10$ agar medium. Colonies were counted 28 days after incubation at $37^{\circ} \mathrm{C}$.

Histopathological analysis. The upper left lobe of each mouse lung was fixed in $10 \%$ formalin, washed in PBS for $24 \mathrm{~h}$, dehydrated in a graded ethanol series, embedded in glycol metacrylate resin (Historesin Embedding Kit; Leica, Heidelberg, Germany) and then sectioned for light microscopy. Sections ( $3 \mu \mathrm{m}$ each) were stained with haematoxylin \& eosin. The sections were analysed using a Leica DMLB 80 microscope connected to a Leica DC300FX camera.

Cytokine evaluation. Two weeks after the last DNA dose, animals were sacrificed and splenic cells were collected and adjusted to $5 \times 10^{6}$ cells $/ \mathrm{ml}$ in RPMI 1640 medium supplemented with $5 \%$ FCS, $20 \mathrm{~mm}$ glutamine and $40 \mathrm{IU} / 1$ of gentamicin. The cells were cultured in 48-well flat-bottomed culture plates (Nunc, Life Tech. Inc., Maryland, MA, US) in the presence of $40 \mu \mathrm{g} / \mathrm{ml}$ of Concanavalin A (ConA). Cytokine levels in culture supernatants were evaluated $48 \mathrm{~h}$ later by ELISA. Cytokines were measured according to the manufacturer's instructions (PharMingen). Briefly, purified monoclonal antibodies, anti-IFN- $\gamma$ (R4-6A2) and anti-IL-5 (TRKF5) were used at $1 \mu \mathrm{g} / \mathrm{ml}$ as capture antibodies. The following biotinylated antibodies were used for detection: antiIFN- $\gamma$ (XMG1.2) and anti-IL-5 (TRFK4) at $0.5 \mu \mathrm{g} / \mathrm{ml}$. Cytokines were also evaluated in the lung homogenates 4 weeks after challenge with $M$. tuberculosis, i.e, at the same time as CFU were determined in the lungs.

Statistical analysis. All figures express the mean \pm SEM for each variable. Statistical analysis was performed using unpaired $t$-test. Values of $P<0.05$ were considered statistically significant.

\section{Results}

\section{Neonatal BCG immunization primes for a strong immune response to pVAXhsp65 in the adult phase}

Immunization of neonate BALB/c mice (5 days old) with BCG did not induce anti-hsp65 antibody production (not shown). Administration of two pVAXhsp65 doses to adult mice by the intramuscular route induced significant levels of antigen-specific IgG1 and IgG2a. Both isotype levels were significantly increased if the animals were previously primed with BCG in the neonatal period. Increased levels of $\mathrm{IgG} 1$ and $\mathrm{IgG} 2 \mathrm{a}$ can be observed in Fig. $1 \mathrm{~A}$ and $\mathrm{B}$, respectively. Inoculation of the empty vector (control) did not induce anti-hsp65 antibodies in animals previously primed or not with BCG.

\section{Neonatal BCG immunization followed by DNAhsp65 boosters in the adult phase is not protective in experimental tuberculosis}

Immunization with BCG in the neonatal period induced protective immunity that remained during adult life. This was clearly demonstrated by a significant decrease in the number of CFU in the lungs of these animals, 30 days after challenge with $M$. tuberculosis. However, the animals that received two boosters with pVAXhsp65 in

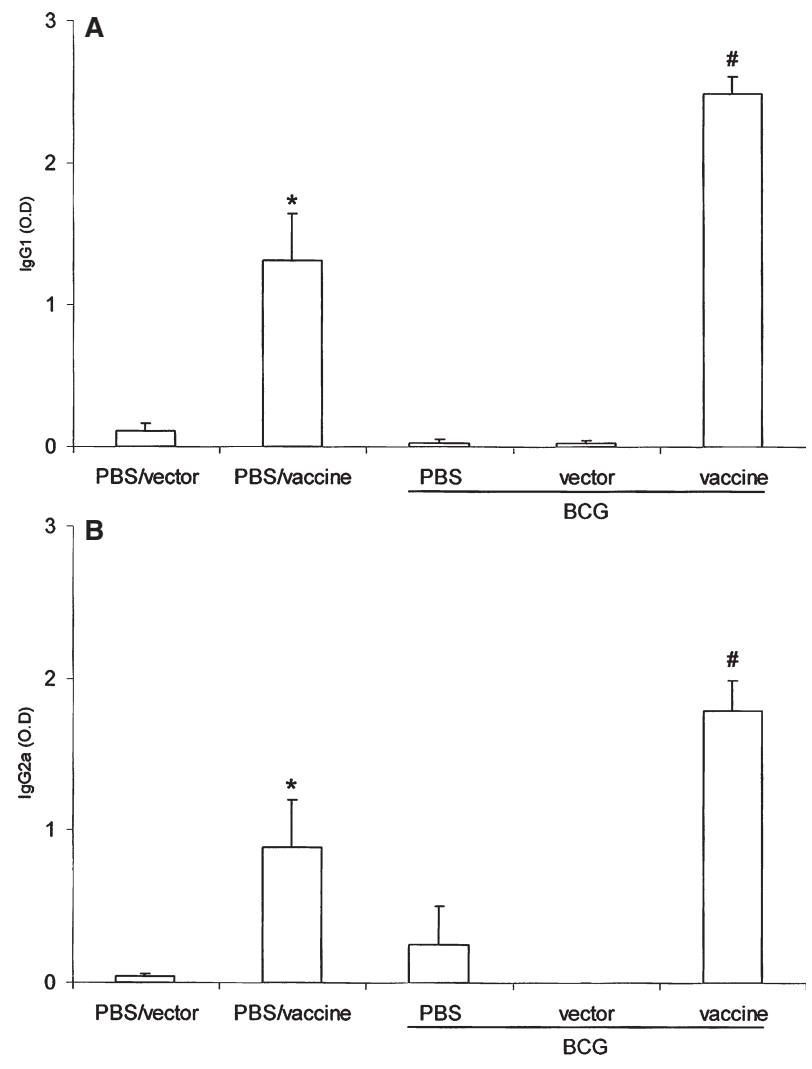

Figure 1 Effect of BCG priming on antibody induction by pVAXhsp65 immunization. Neonate mice ( 5 days old) were immunized in the base of the tail with BCG $\left(10^{5}\right.$ bacilli). Four weeks later, in the adult phase, they received two pVAXhsp65 doses, delivered 2 weeks apart. Two weeks after last DNA dose, IgG1 (A) and IgG2a (B) anti-hsp65 antibody levels were determined by ELISA in blood samples diluted 1:25. Results are presented as mean \pm SEM from 5 to 8 mice per group. $* P<0.05$ in comparison with corresponding control (PBS/Vector); \#P<0.05 in comparison with PBS/Vaccine. 
the adult phase lost this protective immunity. Interestingly, the empty vector (pVAX) triggered a similar deleterious effect, abolishing the protective ability of BCG. These results are shown in Fig. 2A. The control group that was inoculated with two DNAhsp65 doses in the adult phase showed no protection (non-immunized control: $5.88 \log$ CFU per lung; two DNAhsp65 doses: $6.14 \log$ CFU per lung). Lung histopathological analysis showed a very well preserved parenchyma in BCG-vaccinated mice (Fig. 2D), very similar to the structure found in normal animals (Fig. 2B). As expected, infected nonimmunized control animals showed a striking alteration of the parenchyma characterized by intense inflammatory infiltrates dominated by mononuclear cells (Fig. 2C). In animals that received DNAhsp65 boosters, the parenchyma was similar to the control group (infected and without previous immunization) i.e., presented a clear and abundant inflammatory process (Fig. 2E).

\section{Cytokine modulation by DNA in mice primed with BCG in the neonatal period}

This aspect was initially evaluated by production of IFN$\gamma$ and IL-5 in spleen cell cultures from immunized animals stimulated with ConA and later, in mice challenged with mycobacteria, by quantification of these two cytokines in lung homogenates. Administration of pVAXhsp65, or the control vector, to mice previously immunized with BCG did not affect their ability to produce IFN- $\gamma$ in response to ConA stimulation. However, this procedure clearly increased IL- 5 production of spleen cells stimulated with ConA. These results can be
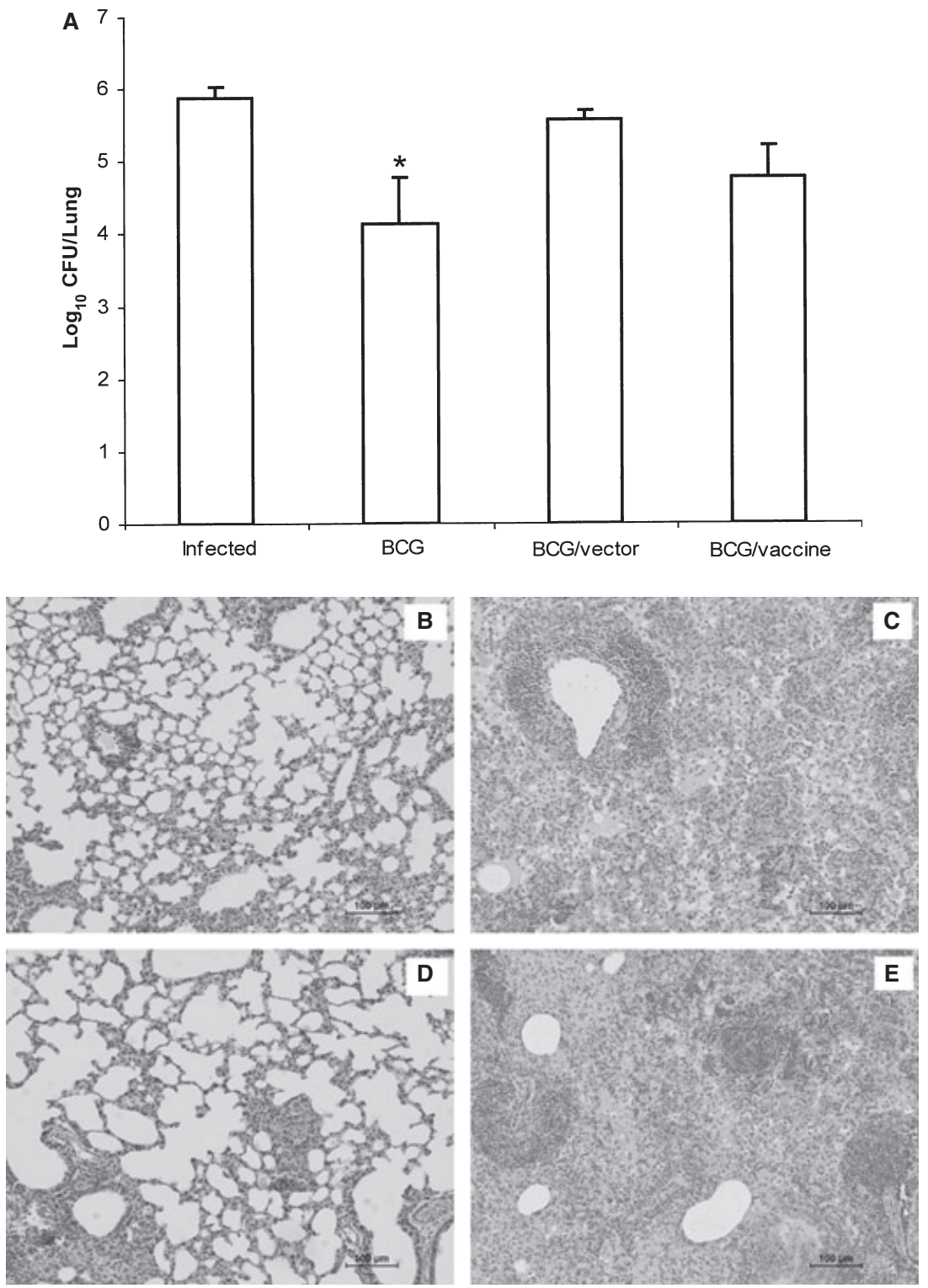

Figure 2 Effect of pVAXhsp65 booster on the protective efficacy of BCG. Neonate mice ( 5 days old) were immunized in the base of the tail with BCG $\left(10^{5}\right.$ bacilli). Four weeks later, in the adult phase, they received two pVAXhsp65 doses, delivered 2 weeks apart. Two weeks after last DNAhsp65 dose the animals were infected with $10^{5} \mathrm{CFU}$ of $M$ tuberculosis $\mathrm{H} 37 \mathrm{Rv}$ by intratracheal route. Four weeks later, the number of live bacteria in the lungs was determined and expressed as Log 10 CFU/lung (A) and the lung parenchyma structure was analysed in $\mathrm{H} \& \mathrm{E}$ stained lung sections: normal mice (B); non-immunized infected mice (C); BCG immunized infected mice (D); BCG/pVAXhsp65 immunized infected mice (E). CFU results are presented as mean \pm SEM from 4 to 7 mice. $* P<0.05$ in comparison with nonimmunized control. 
observed in Fig. $3 \mathrm{~A}$ and $\mathrm{C}$, respectively. No differences were observed in the levels of IFN- $\gamma$ in the lung homogenates (Fig. 3B). However, similar to the results found in the spleen, higher IL-5 levels were found in lung homogenates from groups that received DNA boosters (pVAXhsp65 and pVAX) after BCG immunization in comparison with BCG alone (Fig. 3D). Levels of IFN- $\gamma$ and IL-5 produced by spleen cell cultures (3850 and $240 \mathrm{pg} / \mathrm{ml}$, respectively) and present in lung homogenates (5500 and $245 \mathrm{pg} / \mathrm{ml}$, respectively) in mice immunized with two DNAhsp65 doses were similar to their respective controls.

\section{Discussion}

In the current study, by using an experimental tuberculosis model, we investigated the immunogenicity and the protective efficacy of a prime-boost strategy employing BCG and pVAXhsp65. Neonate mice were initially primed with BCG and then, in the adult phase, boosted twice with a DNA vaccine containing the hsp65 gene from M. leprae (pVAXhsp65). Animals immunized only with pVAXhsp65 produced both, IgG2a and IgG1 anti-hsp65 antibodies, suggesting that this vaccine was inducing a mixed pattern (Th1/Th2) of response. This assumption was based on literature reports that correlate IgG2a and IgG1 with the predominance of Th1 and Th2 responses, respectively. Priming with BCG in the neonatal period, before administration of the DNA vaccine, did not change this mixed profile but clearly improved immunogenicity, determining a significant increase in the production of both isotypes. In the light of these observations, our results suggested that BCG priming in the neonatal period was not skewing the immune response to Th1 or Th2 but instead, it was simply priming for a robust immune response later in the adult phase.

From previous experience with this genetic vaccine, we know that the immunity induced by two doses delivered by the intramuscular route is not protective in experimental tuberculosis. Based on this and the fact that BCG increased pVAXhsp65 immunogenicity, we then tested the protective efficacy of BCG / pVAXhsp65 association. Neonatal BCG vaccination, as was expected from the literature reports, was able to protect the mice challenged with $M$. tuberculosis by the intratracheal route. This protection was characterized by a significant reduction in the number of CFU in animals that were previously vaccinated with BCG. Histopathological analysis also sustained BCG protective ability by showing a very well-preserved lung parenchyma with no signs of inflammation. However, animals that received boosters with pVAXhsp65 or with the empty vector itself in the adult phase lost the protective immunity determined by BCG against $M$. tuberculosis. This was established by the recovery of similar CFU numbers in the lungs of these animals and the non-immunized ones. Also, the lung parenchyma in both, vector and vaccine-boosted animals, was similarly affected, i.e., they presented clear inflammatory infiltrates.
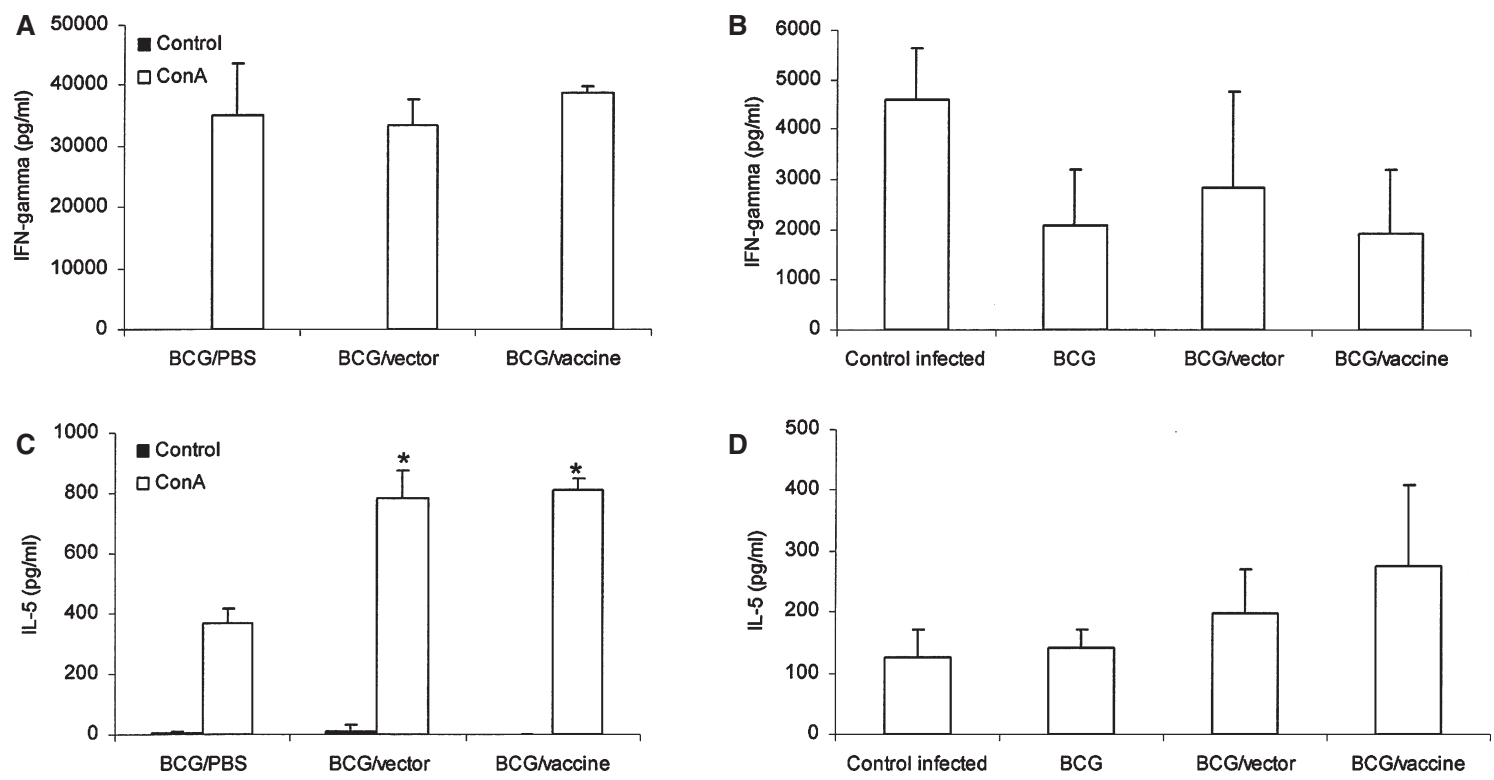

Figure 3 Boosters with DNA (pVAX or pVAXhsp65) increase IL-5 production in mice neonatally primed with BCG. Neonate mice were immunized with BCG and boosted, in the adult phase, with two DNA doses (pVAX or pVAXhsp65). Two weeks after last DNA dose, the animals were tested for IFN- $\gamma(3 \mathrm{~A})$ and IL-5 (3C) production by splenic cells or infected with $M$. tuberculosis. Four weeks after infection, the animals were sacrificed and concentration of IFN- $\gamma(3 \mathrm{~B})$ and IL-5 (3D) was determined in lung homogenates. Results are presented as mean \pm SEM from 4 to 8 mice. $* P<0.05$ in comparison with BCG/PBS group. 
A direct comparison of our results and other publications is hampered by the diversity of prime-boost protocols employed to evaluate protection in tuberculosis. The work of Skinner et al. [19], for example, differently from our findings, showed that neonatal calves primed at birth with BCG vaccine and later boosted with DNA vaccines containing hsp65, hsp70 or Apa, showed enhanced protection against bovine tuberculosis. Even though some authors alerted for the relevance of the immunization sequence [20], Skinner et al. [19], observed that this aspect was not critical. Similarly to our results, Majlessi et al. [21] described increased immunogenicity but not increased protection by using a prime-boost protocol.

An attractive possibility to explain the failure of this prime-boost protocol is that BCG is determining both, priming for hsp immune response and also providing a mixed (Th1 + Th2) microenvironment for the ensuing immune response. In this scenario, the immune response induced by pVAXhsp65 vaccination would be a mixed and strong immune response against hsp65. The antibody results observed in this work point to this possibility and it is also highly supported by various reports. Qazi et al. [22] recently published that BCG primed the immune system for the induction of immunity against a variety of heat shock proteins (hsp) including hsp65. Interestingly, analysis of immunoglobulin isotype profiles and IFN- $\gamma$ production induced against hsps revealed induction of a mixed Th1 / Th2 response. The literature also supports the view that $\mathrm{BCG}$ immunization equally establishes mixed Th1/ Th2 microenvironments in adult and neonate mice [23]. Interestingly, although BCG induced a potent Th1-type response to mycobacterial antigens, it promoted the production of both Th1- and Th2-type cytokines in response to unrelated vaccines [24]. Another possibility that needs to be considered relates to the BCG dose used to prime the neonate mice. As BCG dose defines the Th1 / Th2 nature of the immune response [25], it would be very convenient to evaluate the effect of lower BCG doses in this protocol. As prime-boost strategies represent a relatively new approach in vaccinology, factors as interval between priming and boosting must also be considered, as has been recently discussed [26, 27]. Another interesting explanation for this failure is the possible induction of $\mathrm{T}$ regulatory cells by a combination of BCG with DNAhsp65. Both BCG and hsp65 have been described as potent immunomodulators to control inflammation associated with allergy and autoimmune diseases [28, 29]. In support of this hypothesis, we recently observed that the genetic vaccine containing the hsp65 gene from $M$. leprae was able to control inflammation in experimental diabetes [30, 31].

One of the most intriguing findings of this investigation was the fact that both, vector and vaccine, equally decreased BCG protective ability. This strongly indicates that non-coding sequences of the vector were somehow interfering with the immunity established by BCG vaccination. To try to explain the mechanism involved in this deleterious effect triggered by DNA inoculation, we compared the cytokine profile in BCG and BCG / DNA experimental groups. Splenic cells from BCG, BCG / vector and BCG / vaccine groups produced similar IFN$\gamma$ levels after ConA stimulation. However, groups boosted with pVAX or pVAXhsp65 produced significantly higher IL-5 amounts. Interestingly, lung homogenates from $\mathrm{BCG} /$ vector and $\mathrm{BCG} /$ vaccine groups also showed higher IL-5 levels. Even though these results are not completely enlightening, they suggest that the vector itself is deleteriously modulating the protective immunity established by BCG. It is well accepted that tuberculosis control requires a Th1 type of response which can be compromised by a Th2 response $[32,33]$. We suggest, therefore, that the vector is somehow accentuating the Th2 profile. This possibility is, at least partially, supported by literature reports. For example, unmethylated $\mathrm{CpG}$ motifs that are prevalent in bacteria, including in bacterial plasmids used as vectors for genetic vaccines, are able to enhance both innate and specific (humoral and cellular) immunity via TLRs [34]. It is also described that oligodeoxynucleotides lacking $\mathrm{C}_{\mathrm{pG}}$ dinucleotides mediate Toll-like receptor 9 dependent Th2 biased immuno stimulation [35]. Even though the $\mathrm{CpG}$ content of plasmid vectors is not completely described, we and other authors showed evidences that non-coding plasmid DNA was able to inhibit autoimmunity processes mediated by Th1 cells in experimental diabetes and encephalomyelitis [31, 36-38].

In conclusion, our results demonstrated that priming of newborn mice with BCG followed by pVAXhsp65 boosters later in adult life significantly increased immunogenicity of this genetic vaccine. However, this kind of strategy did not determine protection in experimental tuberculosis. The results also showed that boosters with both, pVAXhsp65 and pVAX (empty vector) abrogated the BCG protective ability. Further studies are necessary to elucidate this negative effect mediated by the vector itself. This investigation would allow elimination of this negative effect and probably to reveal the possible positive role of vaccine in a similar prime-boost strategy.

\section{Acknowledgment}

The authors are grateful to Secretaria da Saúde do Estado de São Paulo for providing BCG and Fundação de Amparo à Pesquisa do Estado de São Paulo (FAPESP) that supported this study with a grant (Proc. No. 03/06348-7).

\section{References}

1 Flynn JL, Chan J. Tuberculosis: latency and reactivation. Infect Immun 2001;69:4195-201. 
2 Rook GA, Hernandez-Pando R. The pathogenesis of tuberculosis. Anпu Rev Microbiol 1996;50:259-84.

3 Andersen P, Doherty TM. The success and failure of BCG-implications for a novel tuberculosis vaccine. Nat Rev Microbiol 2005;3:656-62.

4 Colditz GA, Brewer TF, Berkey CS et al. Efficacy of BCG vaccine in the prevention of tuberculosis. Meta-analysis of the published literature. JAMA 1994;271:698-702.

5 Springett VH, Sutherland I. A re-examination of the variations in the efficacy of BCG vaccination against tuberculosis in clinical trials. Tuber Lung Dis 1994;75:227-33.

6 Behr MA, Wilson MA, Gill WP et al. Comparative genomics of BCG vaccines by whole-genome DNA microarray. Science 1999;284:1520-3

7 Fine PE. Variation in protection by BCG: implications of and for heterologous immunity. Lancet 1995;346:1339-45.

8 Skeiky YA, Sadoff JC. Advances in tuberculosis vaccine strategies. Nat Rev Microbiol 2006;4:469-76.

9 McMurray DN. A coordinated strategy for evaluating new vaccines for human and animal tuberculosis. Tuberculosis (Edinb) 2001;81: $141-6$.

10 Andersen P. TB vaccines: progress and problems. Trends Immunol 2001;22:160-8

11 Tascon RE, Colston MJ, Ragno S, Stavropoulos E, Gregory D, Lowrie DB. Vaccination against tuberculosis by DNA injection. Nat Med 1996;2:888-92.

12 Tanghe A, D'Souza S, Rosseels $\mathrm{V}$ et al. Improved immunogenicity and protective efficacy of a tuberculosis DNA vaccine encoding Ag85 by protein boosting. Infect Immun 2001;69:3041-7.

13 Griffin JF, Mackintosh CG, Slobbe L, Thomson AJ, Buchan GS. Vaccine protocols to optimize the protective efficacy of BCG. Tuber Lung Dis 1999;79:135-43.

14 McShane H, Brookes R, Gilbert SC, Hill AV. Enhanced immunogenicity of $\mathrm{CD} 4(+) \mathrm{t}$-cell responses and protective efficacy of a DNAmodified vaccinia virus Ankara prime-boost vaccination regimen for murine tuberculosis. Infect Immun 2001;69:681-6.

15 Wong YL, Sampson S, Germishuizen WA et al. Drying a tuberculosis vaccine without freezing. Proc Natl Acad Sci U S A 2007; 104:2591-5.

16 Barrios C, Brawand P, Berney M, Brandt C, Lambert PH, Siegrist CA. Neonatal and early life immune responses to various forms of vaccine antigens qualitatively differ from adult responses: predominance of a Th2-biased pattern which persists after adult boosting. Eur J Immunol 1996;26:1489-96.

17 Bonato VL, Lima VM, Tascon RE, Lowrie DB, Silva CL. Identification and characterization of protective $\mathrm{T}$ cells in hsp65 DNA-vaccinated and Mycobacterium tuberculosis - infected mice. Infect Immun 1998;66:169-75.

18 Lowrie DB, Tascon RE, Bonato VL et al. Therapy of tuberculosis in mice by DNA vaccination. Nature 1999;400:269-71.

19 Skinner MA, Wedlock DN, de Lisle GW et al. The order of primeboost vaccination of neonatal calves with Mycobacterium bovis BCG and a DNA vaccine encoding mycobacterial proteins hsp65, hsp70, and Apa is not critical for enhancing protection against bovine tuberculosis. Infect Immun 2005;73:4441-4.

20 Ramshaw IA, Ramsa AJ. The prime-boost strategy: exciting prospects for improved vaccination. Immunol Today 2000;21:163-5.

21 Majlessi L, Simsova M, Jarvis $Z$ et al. An increase in antimicobacterial Th1-cell responses by prime-boost protocols of immunization does not enhance protection against tuberculosis. Infect Immun 2006;74:2128-37.
22 Qazi KR, Qazi MR, Julián E, Singh M, Abedi-Valugerdi M, Fernández $\mathrm{C}$. Exposure to mycobacteria primes the immune system for evolutionarily diverse heat shock proteins. Infect Immun 2005;73:7687-96.

23 Lagranderie M, Balazuc AM, Abolhassani M et al. Development of mixed Th1 / Th2 type immune response and protection against Mycobacterium tuberculosis after rectal or subcutaneous immunization of newborn and adult mice with Mycobacterium bovis BCG. Scand J Immunol 2002;55:293-303.

24 Ota MO, Vekemans J, Schlegel-Haueter SE et al. Influence of Mycobacterium bovis bacillus Calmette-Guérin on antibody and cytokine responses to human neonatal vaccination. $J$ Immunol 2002;168:91925.

25 Power CA, Wei G, Bretscher PA. Mycobacterial dose defines the Th1 / Th2 nature of the immune response independently of whether immunization is administered by the intravenous, subcutaneous, or intradermal route. Infect Immun 1998;66:5743-50.

26 McShane H, Hill A. Prime-boost immunisation strategies for tuberculosis. Microbes Infect 2005;7:962-7.

27 Wong P, Lara-Tejero M, Ploss A, Leiner I, Pamer EG. Rapid development of T cell memory. J Immunol 2004;172:7239-45.

28 Rook GA, Adams V, Hunt J, Palmer R, Martinelli R, Brunet LR. Mycobacteria and other environmental organisms as immunomodulators for immunoregulatory disorders. Springer Semin Immunopathol 2004;25:237-55.

29 van Eden W, van der Zee R, Prakken B. Heat-shock proteins induce T-cell regulation of chronic inflammation. Nat Rev Immunol 2005;5:318-30.

30 Santos RR Jr, Sartori A, Bonato VL et al. Immune modulation induced by tuberculosis DNA vaccine protects non-obese diabetic mice from diabetes progression. Clin Exp Immunol 2007;149:5708.

31 Santos RR Jr, Sartori A, Lima DS et al. DNA vaccine containing the mycobacterial hsp65 gene prevented insulitis in MLD-STZ diabetes. J Immune Based Ther Vaccines 2009;7:4.

32 Rook GA, Hernandez-Pando R. Immunological and endocrinological characteristics of tuberculosis that provide opportunities for immunotherapeutic intervention. Novartis Found Symp 1998;217:7387.

33 Al-Attiyah RJ, Mustafa AS. Mycobacterial antigen-induced T helper type 1 (Th1) and Th2 reactivity of peripheral blood mononuclear cells from diabetic and non-diabetic tuberculosis patients and Мусоbacterium bovis bacilli Calmette-Guérin (BCG)-vaccinated healthy subjects. Clin Exp Immunol 2009;158:64-73.

34 Krieg AM. A role for Toll in autoimmunity. Nat Immunol 2002;3:423-4.

35 Vollmer J, Weeratna RD, Jurk $\mathrm{M}$ et al. Oligodeoxynucleotides lacking $\mathrm{CPG}$ dinucleotides mediate Toll-like receptor 9 dependent $\mathrm{T}$ helper type 2 biased immune stimulation. Immunology 2004;113: 212-23.

36 Boccaccio GL, Mor F, Steinman L. Non-coding plasmid DNA induces IFN-gamma in vivo and suppresses autoimmune encephalomyelitis. Int Immunol 1999;11:289-96.

37 Quintana FJ, Rotem A, Carmi P, Cohen IR. Vaccination with empty plasmid DNA or $\mathrm{CpG}$ oligonucleotide inhibits diabetes in nonobese diabetic mice: modulation of spontaneous $60-\mathrm{kDa}$ heat shock protein autoimmunity. J Immunol 2000;165:6148-55.

38 Zorzella-Pezavento SFG, Chiuso-Minicucci F, França TGD et al. Immunization with pVAXhsp65 decreases inflammation and modulates immune response in experimental encephalomyelitis. Neuroimmunomodulation; (in press). 